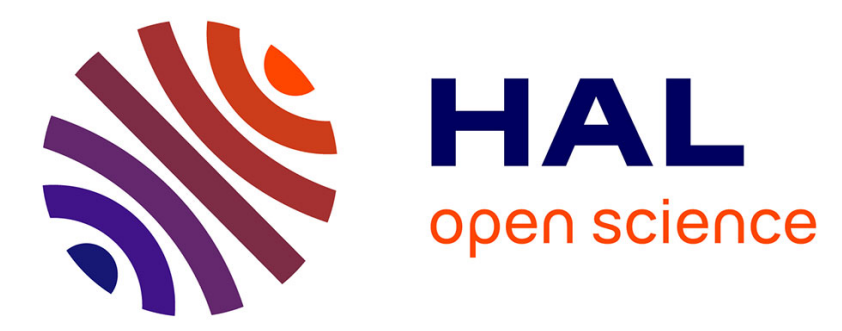

\title{
Intragenome diversity of gene families encoding toxin-like proteins in venomous animals Running title: Diversity of toxin-like gene families
}

\author{
Ricardo C. Rodriguez de La Vega, Tatiana Giraud
}

\section{- To cite this version:}

Ricardo C. Rodriguez de La Vega, Tatiana Giraud. Intragenome diversity of gene families encoding toxin-like proteins in venomous animals Running title: Diversity of toxin-like gene families. Integrative and Comparative Biology, 2016, 10.1093/icb/icw097 . hal-01363004

\section{HAL Id: hal-01363004 \\ https://hal.science/hal-01363004}

Submitted on 9 Sep 2016

HAL is a multi-disciplinary open access archive for the deposit and dissemination of scientific research documents, whether they are published or not. The documents may come from teaching and research institutions in France or abroad, or from public or private research centers.
L'archive ouverte pluridisciplinaire HAL, est destinée au dépôt et à la diffusion de documents scientifiques de niveau recherche, publiés ou non, émanant des établissements d'enseignement et de recherche français ou étrangers, des laboratoires publics ou privés. 


\title{
Intragenome diversity of gene families encoding toxin-like proteins in venomous animals
}

Running title: Diversity of toxin-like gene families

Ricardo C. Rodríguez de la Vega*, Tatiana Giraud

Ecologie Systematique Evolution, UMR8079, CNRS, Univ. Paris-Sud, AgroParisTech, Université ParisSaclay, 91400 Orsay, France

*To whom correspondence should be addressed

Email: ricardo.rodriguezdelavega@gmail.com,ricardo.rodriguez-de-la-vega@u-psud.fr

Tel: +33 169157049, Fax: +33 169154697

\begin{abstract}
The evolution of venoms is the story of how toxins arise and of the processes that generate and maintain their diversity. For animal venoms these processes include recruitment for expression in the venom gland, neofunctionalization, paralogous expansions, and functional divergence. The systematic study of these processes requires the reliable identification of the venom components involved in antagonistic interactions. High-throughput sequencing has the potential of uncovering the entire set of toxins in a given organism, yet the existence of non-venom toxin paralogs and the misleading effects of partial census of the molecular diversity of toxins make necessary to collect complementary evidence to distinguish true toxins from their non-venom paralogs. Here we analyzed the whole genomes of two scorpions, one spider and one snake, aiming at the identification of the full repertoires of genes encoding toxin-like proteins. We classified the entire set of protein-coding genes into paralogous groups and monotypic genes, identified genes encoding toxin-like proteins based on known toxin families, and quantified their expression in both venom-glands and pooled tissues. Our results confirm that genes encoding toxin-like proteins are part of multigene families, and that these families arise by recruitment events from non-toxin genes followed by expansions of the toxin-like protein coding genes. We also show that failing to account for sequence similarity with non-toxin proteins has a considerable misleading effect that can be greatly reduced by comparative transcriptomics. Our study overall contributes to the understanding of the evolutionary dynamics of proteins involved in antagonistic interactions.
\end{abstract}




\section{Introduction}

The remarkable diversity of action and specificity of venoms render them excellent models for studying coevolutionary dynamics. Animal venoms are biological substances usually composed by dozens of active components (a.k.a. toxins [King, 2011]) that are stored and delivered by dedicated cells or organs of one organism to another organism by means of an inflicted wound [Fry et al., 2009; Nelsen et al. 2014]. Toxins are deployed in the extra-organismal space within a context of biotic interactions including predation and defense [Olivera, 2006; Fry et al., 2009]. Venoms have arisen and evolved independently at least 30 times across animal phyla and show remarkable diversity in specificity and action both across and within lineages, which is entirely attributable to the variety of component toxins [Casewell et al., 2013]. This diversity has both phylogenetic and functional components, i.e. reflecting the history of the lineage of the organism producing the venom and the specific biotic interactions in which venom use is/has been a central component. Despite the substantial number of independent evolutionary origins of venom, the high number $(>100,000)$ of extant animal species that use venom as a trophic or defense strategy, and the obvious non-homology of the structures used for venom deployment, animal venoms are built from a limited number of protein families and target a limited number of molecular receptors in the envenomed organisms [Fry et al., 2009]. The interaction between venomous animals and their targets (whether prey or foe) thus represents a narrow molecular "battlefield" on both sides. The high number of independent biological replicates involved in similar coevolutionary interactions involving similar (often orthologous) sets of genes provides a unique opportunity for the rigorous testing of (co)evolutionary hypotheses about adaptation, constraints and convergence, both at the micro- and macroevolutionary scales.

Evolution of animal venoms has been explained in the light of three concurrent paradigms: 1) the "proteome-to-venome" model of protein birth, which postulates independent neo- or subfunctionalization events from various "normal" proteins (i.e. with no extra-organismal selected effect) at the origin of venom proteins [Moura-da-Silva et al., 1996; Fry and Wuster, 2004]; 2) the model of "convergent recruitment" of the same few protein folds into the venoms of phylogenetically distantly related species; in other words, independently evolved venoms are built upon convergent neo- or subfunctionalization of a small set of homologous genes [Duda and Palumbi, 1999; Fry et al., 2009], and; 3) an antagonistic interaction-driven "evolutionary arms-race" scenario of venom-protein evolution leading to hyperdiversified sets of homologous genes [Kordis and Gubensek, 2000; Barlow et al., 2009] (but see [Holding et al., 2016]). These paradigms were tremendously helpful when high-throughput screenings and sequencing became routine analyses. However, two main pitfalls prevented optimal inferences about the processes of evolution of toxins, namely: i) the existence of toxin-like genes with no extra-organismal 
selected effect, e.g. genes encoding toxin-like proteins not expressed in the venom glands [Hargreaves et al., 2014a; Junqueira-de-Azevedo et al., 2015; Reyes-Velasco et al., 2015), and ii) the misleading effects of partial census of the intragenome molecular diversity of toxin-homologs, e.g. failure to sample nontoxin paralogs of genuine toxins from a given organism may erroneously make toxins look as monophyletic in gene trees, thus misleading the inferred recruitment scenario [Casewell et al., 2012]. These issues call for a better reference for expression profiles of toxin-paralogs and a better understanding of intragenome evolutionary dynamics to refine the evolutionary insights that can be drawn from the recent proliferation of venom surveys (e.g. [Undheim et al., 2014; von Reumont et al., 2014; Macrander et al., 2015; Rachamim et al., 2015; Phuong et al., 2016]).

Here we surveyed toxin-homologs and constructed clusters of paralogous genes in four venomous organisms for which whole genomes were available but not systematically mined yet for toxin-like gene content: the scorpions Centruroides exilicauda, from the i5K Initiative [i5K Consortium, 2013], and Mesobuthus martensii [Cao et al., 2013], the spider Acanthoscurria geniculata [Sanggaard et al., 2014], and the snake Ophiophagus hannah [Vonk, Casewell, et al., 2013]). We compared the toxin homologs against reference proteomic profiles to estimate the coverage of gene models on the venom proteomes and mapped available RNAseq reads onto the gene models of M. martensii and $O$. hannah to quantify and compare the expression of toxin paralogs in venom glands and pooled tissues. We discuss our results in terms of the paralogous groups of genes encoding toxin-like proteins.

\section{Methods}

Whole genome sequences and gene models

Draft genomes of the Mygalomorphae spider Acanthoscurria geniculata (GCA_000661875.1), the Buthidae scorpions Centruroides exilicauda (GCA_000671375.1) and Mesobuthus martensii (GCA_000671375.1), and the Elapidae snake Ophiophagus hannah (GCA_000516915.1) were downloaded from NCBI's assembly pages (Table 1). Gene models as amino acid and CDS sequences were downloaded from public repositories as follows: C. exilicauda Primary Gene Set BCM_version_0.5.3 from the corresponding project page at https://i5k.nal.usda.gov/; M. martensii from the whole genome sequencing project at http://lifecenter.sgst.cn/main/en/scorpion.jsp (accessed July 2015), and $O$. hannah from NCBI's assembly page. Translated amino acid sequences of $A$. geniculata gene models were obtained from a previous study [Sanggaard et al., 2014]. Gene models of a Malayan $O$. hannah venom gland transcriptome [Tan et al., 2015] were obtained with the transcripts_to_best_scoring_ORFs.pl script of Transdecoder (http://transdecoder.github.io/) run on contigs obtained from shotgun transcriptome assembly (GenBank accession number: GDRF00000000). 


\section{Identification of genes encoding toxin-like proteins}

We blastp-ed (version 2.2.29+ [Altschul et al., 1997]) all gene models from the four species $(157,164$ gene models in total), against a CD-hit [Li and Godzik, 2006] reduced (-c 0.9 option) SwissProt release 201506 [UniProt Consortium, 2015] protein set (313,332 entries), including the entire set of UniProt reviewed proteins known or predicted to be present in the venoms $(6,341$ proteins, see [Jungo et al., 2012]). We applied two thresholds to identify significant matches: e-value of at most 1e-5 and a minimum of $50 \%$ shortest sequence length coverage. We classified gene models matching known toxins with a simplified "family" level classification based on VenomZone annotation (http://venomzone.expasy.org/). We distinguished between genes encoding toxin-like proteins with best blast hits among VenomZone entries from the same order, which we called VZsame (standing for VenomZone best blast hit in the same taxonomic order), those with best blast hits among VenomZone entries but from a different taxonomic order, which we called VZdiff, and those with best blast hits not among VenomZone entries, which we called SPbest (standing for SwissProt best blast hit). A file relating UniProt identifiers, simplified family classification, including brief description of our simplified classification scheme, is provided as Supplementary File 1.

\section{Construction of putative paralogous groups}

We constructed putative paralogous groups by Markov clustering [van Dongen, 2000; Enright et al., 2002] of blastp searches of gene models for each species against themselves with -evalue option set to 1e10. Clustering was performed with OrthoMCL1.4 with default parameters [Li et al., 2003]. Expression of toxin-like protein-coding genes in venom-gland and non-venom gland tissues Reads from venom-gland and non-venom gland tissues of M. martensii (venom tissues: SRX1376145 and SRX1375230; mixed tissues: SRX1377585) and O. hannah (venom gland: SRX365143; pooled organs: SRX365144) were downloaded from NCBI's Sequence Read Archive (SRA). Mixed tissues reads of $M$. martensii were derived from total RNA extraction of the whole body of a single adult specimen [Cao et al., 2013]. Pooled-organs reads of $O$. hannah were obtained from a multitissue bank including heart, lung, spleen, brain, testes, gall bladder, pancreas, small intestine, kidney, liver, eye, tongue, and stomach [Vonk, Casewell, et al., 2013]. Mapping on gene models was performed with tophat2 [Kim et al., 2013] and fragments per kilobase of gene model per million of reads mapped (FPKM) quantified with cufflinks [Trapnell et al., 2012].

\section{Results and Discussion}

General survey of toxin-like protein coding genes in venomous animal genomes 
In order to identify toxin-like protein-coding genes in the four genomes, we queried the entire predicted proteomes against SwissProt release 2015_06 to which we adjusted all the reviewed sequences known or predicted to be present in animal venoms (as part of UniProt's toxin annotation program VenomZone [Jungo et al., 2012]). Sequence comparisons against annotated venom proteins revealed that each genome encoded hundreds of toxin-like proteins, belonging to at least 14 and up to 29 VenomZone families, in $C$. exilicauda and $O$. hannah, respectively.

With our annotation scheme, we identified 375 A. geniculata gene models with significant hits among VenomZone entries. The number of genes inferred in A. geniculata to encode toxin-like proteins was smaller than previously estimated by similar annotation transfer to gene models derived from high throughput RNAseq of venom glands for Mygalomorphae spiders, e.g. 626 "toxin precursors" in Ornithoctonus huwena [Zhang et al., 2014] and 1,136 "potential toxin precursors" in Haplopelma hainanum [Zhang et al., 2015]. While a combined venom-gland transcriptomics and proteomics confirmed only 120 proteins in A. geniculata venom [Sanggaard et al., 2014], these confirmed venom proteins were more likely to match VenomZone protein families within spiders than expected by chance (Chi-square with Yates correction p-value $<0.0001$ ).

The 339 C. exilicauda gene models that encoded scorpion toxin-like proteins represented a 3-4 fold larger set than the venom components estimated from two closely related species: 72 venom-gland enriched gene models matching known toxins were recovered from deep sequenced venom gland transcriptomics of Centruroides noxius [Rendon-Anaya et al., 2012] and 104 venom components identified by mass fingerprinting of Centruroides tecomanus venom [Valdez-Velazquez et al., 2014]. Comparison of the full set of of protein-coding genes against re-assembled gene models of $C$. noxius (SRAs: SRX115875, SRX115901 and SRX115902) revealed that most C. exilicauda genes encoding toxin-like proteins (246/339) had significant blast hits to a total of 304 re-assembled gene models expressed in the venom glands of $C$. noxius [Rodríguez de la Vega et al., unpublished]. Although the number of Mesobuthus martensii genes encoding toxin-like proteins (273) was closer to the number of experimentally-confirmed venom proteins in the same species [Xu et al., 2014], we could match only 68 gene models from the whole genome sequencing because the identification of most venom proteins in this previous publication were based on an unpublished transcriptome assembly. Nonetheless, re-mapping of transcriptome reads confirmed that $57 \%$ of the genes encoding toxin-like proteins $(172 / 304)$ were expressed in the venom glands of M. martensii (see below).

According to our sequence similarity searches, the genome of the elapid $O$. hannah encoded 351 toxin-like proteins, out of which nine matched known three-finger toxins (3FTx), which are paradigmatic venom components of elapids [Utkin, 2013]. This result stood in sharp contrast with three available 
proteomic surveys of the venom from the same species: Vonk, Casewell et al., [2013] identified 82 proteins in the venom, with 31 unique 3FTx representing the largest group; Petras et al., [2015] identified over 100 proteins in the venom by a combined proteomic approach, among which 47 corresponded to unique 3FTx; and finally, while less proteins were identified in the venoms of one Malayan specimen by Tan et al., [2015], the proportion of unique 3FTx (13/45) was in line with the other reports. When applying the same blast thresholds (e-value $<=1 \mathrm{e}-5$ and $50 \%$ query length) to the full set of contigs obtained from the venom gland transcriptome Malayan specimen (29,975 in total [Tan et al., 2015]), only 10 contigs matched 3FTx; for nine of them, their best blast hit within whole genome sequencing derived gene models corresponded precisely to the ones we annotated as 3FTx.

Whereas it is a common practice to annotate a gene model as a putative toxin if sequence similarity similarity is found within dedicated databases (e.g. [Prashanth and Lewis, 2015; Rachamim et al., 2015; Junior et al., 2016; Verdes et al., 2016]), some of them may be genes without a toxin role and not secreted in venoms. In order to filter these out, we queried the entire UniProt for identifying, among the set of genes showing similarity to toxin-encoding genes, the genes that were more similar to other genes with non-venom related functions. We found that, for the spider A. geniculata and the snake $O$. hannah, a majority of the gene models with significant similarity with VenomZone entries from the same taxonomic order also had significant similarity with proteins not known to be expressed in the venom glands of the corresponding orders (Table 2). For example for A. geniculata, 245 out of the 375 gene models with significant similarity to known spider toxins matched non-venom proteins with better expect values. As the queried database was the same, the lower the blast e-value, the more significant the similarity is. Prompted by this observation, we classified gene models encoding toxin-like proteins as: VZsame, for those whose best blastp match was a VenomZone entry from the same taxonomic order; VZdiff, for those whose best blastp match was a VenomZone entry from a different taxonomic order; SPbest, for those whose best blastp match was not a VenomZone sequence. Figure 1 shows as stacked bars the relative fractions of these three categories for each species.

\section{Few protein families dominate toxin-like proteins}

While our annotation transfer scheme identified hundreds of toxin-like proteins in each of the analyzed genomes, their sequence diversity was notwithstanding largely restricted to a handful of protein families. In all cases the five most common VenomZone (i.e. toxin) families accounted for $\sim 70 \%$ of the genes encoding toxin-like proteins, suggesting that that these genes form large multi-gene families. This was further supported by the finding that genes encoding toxin-like proteins were more likely to belong to paralogous groups than any other gene model in the genomes (Table 2, Chi-square with Yates correction 
p-value $<0.001$ for all four species). For example, $88 \%$ (298/339) of genes encoding toxin-like proteins in the C. exilicauda genome were clustered into 28 paralogous groups, whereas the proportion of gene models belonging to any of the 2,524 paralogous groups was less than $60 \%$ for the entire genome $(17,885 / 30,465)$. The median size of paralogous groups containing genes encoding toxin-like proteins ranged from four for A. geniculata to seven for M. martensii, while the median size in the entire set of paralogous genes was two for $A$. geniculata and three for the other species (Supplementary Figure 1). The number of paralogous groups with at least one gene encoding a toxin-like protein, regardless of their VenomZone match-based classification, was 60 for A. geniculata, 50 for C. exilicauda, 63 for $M$. martensii, and 54 for $O$. hannah. Thus, the expected complexity of the venoms is largely defined by a limited number of paralogous groups.

In the spider A. geniculata, the VenomZone protein family with the largest number of matching gene models was Latrotoxin, with 72 sequences belonging to 12 paralogous groups and 5 singletons. This was unexpected because latrotoxins have only been identified directly in the venom of Latrodectus species so far. Homologous sequences have however been found in other spiders from the same Theridiidae family [Garb and Hayashi, 2013] and several partial transcriptome-based gene models are moderately expressed in the venom gland of the theridiid Steatoda grossa [Haney et al., 2016]. In our dataset of $A$. geniculata, gene models with significant similarity with Latrotoxin sequences were partial matches to the $\mathrm{C}$-terminal part containing ankyrin-repeats, and also matched other ankyrin-repeat containing proteins in UniProt, with even better e-values (i.e. the gene models were classified as SPbest). As no large molecular weight proteins resembling Latrotoxins have ever been reported in Mygalomorphae spiders, we suspect that these gene models encode non-toxin proteins. Other VenomZone protein families with large numbers of matches among $A$. geniculata gene models were proteases from three different families (Peptidase PS1 and Metalloproteases M12A and M13) and Kunitz-type proteins, likely with derived functions. However, most of the gene models were classified as SPbest (i.e. they matched non-venom proteins with better blast e-values). Considering only gene models with best blastp hits within VenomZone proteins from spiders (i.e. gene models classified as VZsame), the top-five families were: Metalloprotease M12A, Acetylcholinesterase, Knottin (defined by the presence of an Inhibitory Cystine Knot, ICK, motif), Kunitz-type proteins and Metalloprotease M13. Spider venoms are known to be dominated by small disulfide-rich proteins [King and Hardy, 2013], among which Kunitztype proteins and ICK motif containing proteins are the most common types in Mygalomorphae venoms [Tang et al., 2010; Borges et al., 2016]. In the A. geniculata genome we found only 10 gene models encoding ICK peptides (in 3 paralogous groups and 6 singletons), which is considerably smaller than the number of "protoxins" identified by Sanggaard et al., [2014] in their merged transcriptomes, but close to the eight verified in the venom proteome. Because gene calling may be tricky for ICK-containing 
proteins, as these tend to be rather short and extremely similar, the assembly procedure may have merged together bona fide genes into a single gene model. Proteomic characterization of $A$. geniculata venom identified proteases as the main components [Sanggaard et al., 2014]. While the function of peptidases and proteases in spider venom has not been formally tested, they are usually considered to assist in the spread of the venom through connective tissue [Kuhn-Nentwig et al., 2011], although a role in venom protein processing seems also likely [Sanggaard et al., 2014]. Interestingly, three gene models (two VZsame) in one paralogous group matched phospholipase D enzymes, also known as sphingomyelinase D, which are the infamous dermonecrotic venom components of sicariid spiders [Binford et al., 2009; Pedroso et al., 2015].

VenomZone family annotations for the two Buthidae scorpions were similar, with more than $80 \%$ of all matched gene models classified as VZsame (Figure 1). Gene models matching sodium channel modulators (NaTx) and Metalloproteases M12B were the two most frequent annotations in both cases. Scorpion sodium channel modulators are $\sim 7 \mathrm{KDa}$ disulfide rich proteins with a Cystine Stabilized alpha/beta (CSab) structural motif. Gene models matching the other main group of CSab proteins from scorpion venoms, the shorter potassium channel blockers (KTx), were also common among VZsame annotations. Both types of proteins are the paradigmatic components of Buthidae scorpion venoms [Rodríguez de la Vega et al., 2010; Smith and Alewood, 2015]. Two protease inhibitors, Kunitz-type and trypsin inhibitory-like (TIL), were also among the most frequent VZsame annotations in both species. Mass spectrometry based proteomics identified KTx and NaTx as the main toxin types in M. martensii venom, collectively accounting for $\sim 16 \%$ of all identified proteins (22 NaTx and $12 \mathrm{KTx}$ ), whereas venom proteases were found in much lower proportion [Xu et al., 2014]. In both scorpions nearly $30 \%$ of all gene models encoding toxin-like proteins had best blast hits with VenomZone entries from a different order (what we classified as VZdiff, see Figure 1), with spider toxins being matched by more than half of these gene models.

Genes encoding toxin-like proteins in the genome of $O$. hannah were more diversified, matching the largest number of different VenomZone families. The top 10 most frequent annotations accounted for almost $75 \%$ of all toxin-like protein coding genes, including two proteases, two carbohydrate binding proteins, and two families with no clear function in venoms (Ohanin and Calmodulin-like). However, nearly $77 \%$ of all gene models encoding toxin-like proteins had higher similarity with non-venom proteins (i.e. what we classified as SPbest). Notably, when we considered only best blast hits with squamate VenomZone sequences, the relative contribution of the different families changed (with two cysteine-rich protein families as part of the top-five, 3FTx and Kunitz-type, but also proteases and one carbohydrate binding protein), but with almost no loss in venom complexity estimated as the number of 
different protein families (Figure 2). In any case, even though we found the typical elapid toxins such as 3FTx and phospholipase A2, this distribution of VenomZone families was remarkably different from the short neurotoxin rich pattern that is considered typical in Elapidae venoms (e.g. [Correa-Neto et al., 2011; Laustsen et al., 2015]). Quantitative proteomics of $O$. hannah previously found metalloproteases as the second most abundant venom component in terms of total protein content, as revealed by gel densitometry of a Malayan specimen ( 30\% [Tan et al., 2015]) or by high performance liquid chromatography of an Indonesian specimen ( 12\% [Petras et al., 2015]). Some elapids are known to have switched from a mainly neurotoxic venom to a mainly cytotoxic one [Tan et al., 2016], but whether the abundance of cytotoxin-like proteins is related to the peculiar trophic strategy of $O$. hannah, with its strong preference for snakes, deserves further investigations.

The genes encoding toxin-like proteins in a range of venomous animals are thus confirmed to be part of a few large protein families [Fry et al., 2009; Casewell et al., 2013]. The question remains, however, of whether the proteins encoded by those genes are true toxins, i.e. whether the active components of the venom evolved in the context of antagonistic interactions. In the search of clues about the origin of genes encoding toxin-like proteins, we then investigated the composition of paralogous groups in terms of the sequence similarity with known proteins.

\section{Non-toxin paralogs of genes encoding toxin-like proteins}

Comparison with known proteins revealed that most of the genes encoding toxin-like proteins in the two Buthidae scorpions had their best blast hit within VenomZone Scorpiones entries (i.e. most are VZsame). In contrast, more than half of the toxin-like proteins predicted in the genomes of A. geniculata and $O$. hannah had best blast hits with proteins not known to be present in the venom of any species (i.e. most were SPbest, see Figure 1). We extended this observation by looking at the paralogous groups containing genes encoding toxin-like proteins. For this, we investigated the relative contribution of VZsame, VZdiff, and SPbest in paralogous groups containing genes encoding toxin-like proteins in the search of evidence, or lack thereof, of paralogous expansions associated to the transition between non-toxin and toxin functions.

The number of paralogous groups with at least one gene encoding a toxin-like protein added up to 60 in A. geniculata, 50 in C. exilicauda, 63 in M. martensii and 54 in O. hannah, although roughly one third of these paralogous groups in the spider and the snake contained no gene models with best blastp hits among VenomZone proteins (Figure 2 and Supplementary File 2). Paralogous groups composed only by gene models matching known toxins with best blastp values (i.e. VZsame or VZdiff categories) accounted for $52 \%$ of the genes encoding toxin-like proteins in C. exilicauda, $20 \%$ in M. martensii, $8 \%$ in 
A. geniculata and less than $4 \%$ in $O$. hannaht. In all four species, paralogous groups containing genes encoding toxin-like proteins were larger than paralogous groups with blast hits only with non-venom proteins (Supplementary Figure s1) and also included genes with no match to any known venom protein or with better blast hits with non-venom proteins (Figure 2). These results reinforce the idea that pervasive gene recruitment followed by paralogous expansions have played an important role during venom evolution [Fry et al., 2009]. However, we note that sequence similarity to known toxins alone does not imply that a given gene model encodes a bona fide toxin. In the search of further evidence that could distinguish true toxins from their non-toxin paralogs, we complemented our inquiries to the genomes of venomous animals by quantifying the expression of genes encoding toxin-like proteins in replenishing venom glands and pooled organs of the Buthidae scorpion M. martensii and the Elapidae snake $O$. hannah.

Relative expression of genes encoding toxin-like proteins in venom glands and pooled organs We could map transcriptome reads on 55\% of the gene models in M. martensii and on $63 \%$ of the gene models in O. hannah (Supplementary Files 3 and 4). Genes encoding toxin-like proteins in M. martensii were more likely to be expressed in at least one of the transcriptome sets than gene models in general (221/273 toxin-like genes with fpkm values greater than one vs 17629/32016 of all gene models, twotailed chi-square with Yates correction $p$-value $<0.0001)$. Although the set of expressed genes was not significantly different in the three sources sampled (78\% of the expressed genes had reads mapped from mixed tissues, resting venom gland and venom gland one week after electric stimulation), we found a significant enrichment of VZsame gene models among those overexpressed in the venom glands (70/172 VZsame vs 2344/32016 all gene models, two-tailed chi-square with Yates correction p-value $<0.0001$, see Figure 3). Most of the overexpressed gene models in venom glands were annotated as the typical scorpion toxins $\operatorname{NaTx}$ (16/32 gene models) and KTx (14/15 gene models), along with 19/32 M12B metalloproteases, closely matching the venom composition of this species as revealed by proteomic profiling [Xu et al., 2014]. Non-toxin paralogs of VZsame gene models with venom gland biased expression were less likely to be overexpressed in the venom glands than expected by chance $(198 / 4189$ non-toxin paralogs of VZsame gene models vs 2344/32016 all gene models in paralogous groups, twotailed chi-square with Yates correction p-value $<0.0001$ ). In summary, genes models predicted to encode toxin-like proteins by simply similarity searches matched known venom composition of $M$. martensii more likely if they are overexpressed in the venom gland and have best blast hits with known scorpion venom proteins. 
For the case of $O$. hannah, the proportion of genes encoding toxin-like proteins being expressed in at least one of the transcriptome sets was slightly greater than the proportion of gene models in general (239/351 toxin-like genes with fpkm values greater than one vs 11541/18445 of all gene models, twotailed chi-square with Yates correction $p$-value $=0.0341$ ). Strikingly, $\sim 60 \%$ of $O$. hannah genes encoding toxin-like proteins with mapped reads were expressed in pooled organs but not in the venom gland, and most of them (129) were classified as SPbest in our annotation scheme (i.e. matching non-venom proteins with better e-values than any VenomZone entry). Conversely, we mapped venom gland transcriptome reads on 94 O. hannah genes encoding toxin-like proteins, with VZsame gene models being more likely to be found expressed in the venom gland than SPbest gene models (45/68 VZsame vs 48/270 SPbest, two-tailed Fisher's exact test, p-value $<0.0001)$, with a clear signal of venom gland overexpression $(33 / 68$ VZsame overexpressed in the venom gland vs 7/270 SPbest overexpressed in the venom gland, two-tailed Fisher's exact test $p$-value $<0.0001$, see Figure 3). In fact, venom gland appeared to be depleted of nontoxin or SPbest paralogs of the 28 venom gland overexpressed VZsame gene models in paralogous groups (28/58 VZsame overexpressed in the venom gland vs $8 / 288$ of non-toxin or SPbest in the same paralogous groups, two-tailed Fisher's exact test p-value $<0.0001)$.

We found no evidence of expression in the venom gland for eight VenomZone squamates protein families for which there is at least one matching gene model in $O$. hannah genome. This was in particular the case for C-type lectins and multi-copper oxidases which are among the most frequent annotations of genes encoding toxin-like proteins and are fairly expressed in the pooled tissues transcriptome (which do not include the accessory gland [Vonk, Casewell, et al., 2013]). Although pooled organs reads mapped on $62 \%$ of all gene models and venom gland reads mapped on only $32 \%$ of the gene models, the difference between the expression profiles of VZsame and SPbest models was not related to sequencing efforts as both transcriptomes had roughly the same size $\left(1.8 \times 10^{7}\right.$ reads from pooled organs vs $1.5 \times 10^{7}$ reads from venom gland) and the mapping rate was similar ( $28 \%$ from pooled organs vs $21 \%$ from venom gland). Despite the lower number of gene models matching 3FTx, the profile of venom gland overexpressed VZsame gene models closely matched the composition revealed by proteomic analysis of $O$. hannah venom [Petras et al., 2015; Tan et al., 2015]). Genes encoding toxin-like proteins and annotated as 3FTx, metalloprotease M12B, calmodulin-like, complement C3 homologs, disintegrins, or peptidase PS1, added up to 18 out of the 33 VZsame gene models with venom gland biased expression. These results are concordant with the ones obtained for the scorpion M. martensii discussed above, i.e. comparative transcriptomes can help to reduce the overestimation of both the number and the diversity of venom components common in unsupervised sequence-similarity based annotation. 
In summary, we have shown that comparison of gene expression between venom glands and nonvenom tissues is instrumental in distinguishing genes encoding proteins likely involved in the venom function from their non-toxin paralogs. Failing to account for this have multiple misleading effects, most notably regarding tests of the recruitment hypothesis (i.e. whether there has been one or multiple recruitment events, see [Casewell et al., 2012; Hargreaves et al., 2014b]) and the adequacy of selection tests performed on alignments merging both old and recent paralogs as these are likely to be influenced by the different evolutionary rates of both (see [Jordan et al., 2004; Aguileta et al., 2006; Pegueroles et al., 2013]) and affected by gene conversion leading to overestimation of positive selection [Casola and Hahn, 2009]. The quantitative differences between venom glands and pooled tissues expression of genes encoding toxin-like proteins in M. martensii and the restricted set of VZsame expressed genes in $O$. hannah were both suggestive of specialized gene expression programs in the venom glands, but we note that further sequencing effort in strictly comparative conditions are needed before this hypothesis can be properly tested.

\section{Concluding remarks}

Venom composition was traditionally estimated by painstaking biochemical separation of precious, often rare, biological material. The advent of high-throughput sequencing and improvements in mass spectrometry based protein identification have prompted a surge on venom surveys that have skyrocketed the number of assumed venom components and greatly enlarged their molecular diversity. Most of these novelties, however, are not based on evidence of biologically meaningful activity, nor is common to find works comparing the expression of genes encoding toxin-like proteins in tissues other than the venom gland (but see [Casewell et al., 2012; Hargreaves et al., 2014a; Junqueira-de-Azevedo et al., 2015; ReyesVelasco et al., 2015]). Here we have shown that genes encoding toxin-like proteins in a range of venomous animals are part of multigene families, that mainly originated by recruitment events from nontoxin paralogs followed by paralogous expansions of the genes encoding toxin-like proteins. However, we have also shown that failing to account for sequence similarity has a considerable misleading effect on the identification of the genes encoding bona fide toxins. Finally, we have shown that comparative transcriptomics is a powerful approach to greatly reduce the misleading effect of unsupervised sequence similarity-based annotation.

\section{Acknowledgements}

RCRV research was funded by a Marie Sklowdowska-Curie / Pres-SUD grant (Cofund No. 246556). This contribution greatly benefited from the discussions carried out at a NESCent catalysis meeting in 2014 
and the SICB annual meeting 2016's symposium on Integrative and Comparative Biology of Venom organized by M. Daly and L. Gibbs.

\section{Figure legends}

Figure 1. Classification of gene models encoding toxin-like proteins in four venomous animals. Gene models were queried against a CD-hit reduced UniProt release from June 2015, to which we adjusted all reviewed proteins known to be present in animal venoms (VenomZone). Gene models with significant hits among known toxins were classified as VZsame if the best blast hit was among VenomZone entries from the same taxonomic order (e.g. gene models from scorpions matching VenomZone entries from the order Scorpiones), or as VZdiff if the best blast hit was among VenomZone entries but from a different taxonomic order, or as SPbest if the best blast hit was a protein not known to be present in the venom of any animal. Species abbreviations: Acaga, Acanthoscurria geniculata; Censc, Centruroides exilicauda; Mesma, Mesobuthus martensii; Ophha, Ophiophagus hannah.

Figure 2. Composition of paralogous groups containing genes encoding toxin-like proteins. Each bar corresponds to a single paralogous group, following the color key in the inset with abbreviated VenomZone family names (see Supplementary File 1 for full names). Other abbreviations: aaSPonly, paralogs of genes encoding toxin-like proteins with no match with VenomZone entries; aaSPbest genes encoding toxin-like proteins with better blast matches among non venom proteins; aaNOshit, paralogs of genes encoding toxin-like proteins with no match against known proteins. Only the largest 25 paralogous groups are plotted and the rest pooled in a single stacked bar (pooledPG). Genes encoding toxin-like proteins not assigened to any paralogous group are plotted together in the bars labeled noOG. The figure was obtained with the "barplot" function of R with annotations parsed from Supplementary File 2. Figure 3. Expression profiles of toxin-like and their paralogs in pooled tissues and venom glands. Scatter plots of $\log (\mathrm{fpkm})$ values according to transcriptome source (PT, pooled tissues; VG resting venom gland; VG active venom gland) and similarity with known toxins (VZsame, SPbest or Sponly/NOhit). Symbols are color-coded based on VenomZone families as in Figure 2. Thin lines delimit the 0.25 and 0.75 quartiles. Bold lines correspond to the median values. The figure was obtained with the "beeswarm" package and "bxplot" function of R.

\section{Supplementary Materials}

Supplementary file 1: VenomZone.txt 
UniProt accession codes, taxonomic order, and abbreviated family names. Comments lines (starting with \#) relate the long and short VenomZone family names.

Supplementary file 2: SpePG_VZ_features.txt

A tab-separated annotation file with all gene models encoding toxin-like proteins and their paralogs. Columns are as follows (see also comment lines): 1) Arbitrary gene model ID, 2) paralogous group ID (noOG: no cluster assigned), 3) UniProt identifier of the best blast hit; 4) Sequence identity; 5) length of the alignment; 6) e-value; 7) Category of the gene model (VZsame, VZdiff, SPbest, SPonly, or NOshit, see text for details); 8) VenomZone family based classification (see Supplementary File 1). NA, not applicable.

\section{Supplementary file 3: Mesma_fpkm.txt}

A tab separated file with fpkm values of all gene models encoding toxin-like proteins and their paralogs in transcriptomes from pooled tissues, resting venom gland and venom gland after one week of electrical stimulation. Columns: 1) Gene model ID, 2) length of the protein, 3) paralogous group ID or noOG; 4) Category of the gene model, 5) VenomZone family based classification, 6) fpkm in pooled tissues; 7) fpkm in resting venom glands, and 8) fpkm in venom glands after one week of electrical stimulation. ND, not detected.

\section{Supplementary file 4: Ophha_fpkm.txt}

A tab-separated file with fpkm values of all gene models encoding toxin-like proteins and their paralogs in transcriptomes from pooled tissues and venom gland. The first five columns are as in Supplementary file 3, column 6) fpkm in pooled tissues, column 7) fpkm in venom gland. ND, not detected. Supplementary figure 1: Paralogous groups with gene models encoding toxin-like proteins are larger than the median in each species. Abbreviations: NO, paralogous groups without any gene encoding a toxin-like protein; VZ, paralogous groups containing at least one gene with significant similarity to VenomZone proteins; Acaga, A. geniculata; Censc, C. exilicauda; Mesma, M. martensii; Ophha, O. hannah. Boxplot obtained with the boxplot function of $\mathrm{R}$ with data parsed from supplementary file 2 .

\section{References}

Aguileta G, Bielawski JP, Yang Z. Evolutionary rate variation among vertebrate beta globin genes: implications for dating gene family duplication events. Gene. 2006;380:21-9.

Altschul SF, Madden TL, Schäffer AA, Zhang J, Zhang Z, Miller W, Lipman DJ. Gapped BLAST and PSI-BLAST: a new generation of protein database search programs. Nucleic Acids Res 1997;25:3389-402.

Barlow A, Pook CE, Harrison RA, Wüster W. Coevolution of diet and prey-specific venom activity supports the role of selection in snake venom evolution. Proc Biol Sci 2009;276:2443-9. 
Binford GJ, Bodner MR, Cordes MH, Baldwin KL, Rynerson MR, Burns SN, Zobel-Thropp PA. Molecular evolution, functional variation, and proposed nomenclature of the gene family that includes sphingomyelinase D in sicariid spider venoms. Mol Biol Evol 2009;26:547-66.

Borges MH, Figueiredo SG, Leprevost FV, De Lima ME, Cordeiro Mdo N, Diniz MR, Moresco J, Carvalho PC, Yates JR. Venomous extract protein profile of Brazilian tarantula Grammostola iheringi: searching for potential biotechnological applications. J Proteomics 2016 16;136:35-47.

Cao Z, Yu Y, Wu Y, Hao P, Di Z, He Y, Chen Z, Yang W, Shen Z, He X, Sheng J, Xu X, Pan B, Feng J, Yang X, Hong W, Zhao W, Li Z, Huang K, Li T, Kong Y, Liu H, Jiang D, Zhang B, Hu J, Hu Y, Wang B, Dai J, Yuan B, Feng Y, Huang W, Xing X, Zhao G, Li X, Li Y, Li W. The genome of Mesobuthus martensii reveals a unique adaptation model of arthropods. Nat Commun. 2013;4:2602.

Casewell NR, Wüster W, Vonk FJ, Harrison RA, Fry BG. Complex cocktails: the evolutionary novelty of venoms. Trends Ecol Evol 2013;28:219-29.

Casewell NR, Huttley GA, Wüster W. Dynamic evolution of venom proteins in squamate reptiles. Nat Commun 2012;3:1066.

Casola C, Hahn MW. Gene conversion among paralogs results in moderate false detection of positive selection using likelihood methods. J Mol Evol 2009;68:679-87.

Corrêa-Netto C, Junqueira-de-Azevedo Ide L, Silva DA, Ho PL, Leitão-de-Araújo M, Alves ML, Sanz L, Foguel D, Zingali RB, Calvete JJ. Snake venomics and venom gland transcriptomic analysis of Brazilian coral snakes, Micrurus altirostris and M. corallinus. J Proteomics 2011;74:1795-809.

Duda TF Jr, Palumbi SR. Molecular genetics of ecological diversification: duplication and rapid evolution of toxin genes of the venomous gastropod Conus. Proc Natl Acad Sci USA 1999;96:6820-3.

Enright AJ, Van Dongen S, Ouzounis CA. An efficient algorithm for large-scale detection of protein families. Nucleic Acids Res 2002;30:1575-84.

Fry BG, Roelants K, Champagne DE, Scheib H, Tyndall JD, King GF, Nevalainen TJ, Norman JA, Lewis RJ, Norton RS, Renjifo C, Rodríguez de la Vega RC. The toxicogenomic multiverse: convergent recruitment of proteins into animal venoms. Annu Rev Genomics Hum Genet. 2009;10:483-511.

Fry BG, Wüster W. Assembling an arsenal: origin and evolution of the snake venom proteome inferred from phylogenetic analysis of toxin sequences. Mol Biol Evol 2004;21:870-83.

Garb JE, Hayashi CY. Molecular evolution of $\backslash \mathrm{u} 03 \mathrm{~b} 1-$ latrotoxin, the exceptionally potent vertebrate neurotoxin in black widow spider venom. Mol Biol Evol 2013;30:999-1014.

Haney RA, Clarke TH, Gadgil R, Fitzpatrick R, Hayashi CY, Ayoub NA, Garb JE. Effects of Gene Duplication, Positive Selection, and Shifts in Gene Expression on the Evolution of the Venom Gland Transcriptome in Widow Spiders. Genome Biol Evol 2016;8:228-42. 
Hargreaves AD, Swain MT, Logan DW, Mulley JF. Testing the Toxicofera: comparative transcriptomics casts doubt on the single, early evolution of the reptile venom system. Toxicon 2014a;92:140-56.

Hargreaves AD, Swain MT, Hegarty MJ, Logan DW, Mulley JF. Restriction and recruitment-gene duplication and the origin and evolution of snake venom toxins. Genome Biol Evol 2014b;6:2088-95.

Holding ML, Biardi JE, Gibbs HL. Coevolution of venom function and venom resistance in a rattlesnake predator and its squirrel prey. Proc Biol Sci 2016;283:20152841.

i5K Consortium. The i5K Initiative: advancing arthropod genomics for knowledge, human health, agriculture, and the environment. J Hered 2013;104:595-600.

Jordan IK, Wolf YI, Koonin EV. Duplicated genes evolve slower than singletons despite the initial rate increase. BMC Evol Biol 2004;4:22.

Jungo F, Bougueleret L, Xenarios I, Poux S. The UniProtKB/Swiss-Prot Tox-Prot program: A central hub of integrated venom protein data. Toxicon 2012;60:551-7.

Júnior NG, Fernandes Gda R, Cardoso MH, Costa FF, Cândido Ede S, Neto DG,Mortari MR, Schwartz EF, Franco OL, de Alencar SA. Venom gland transcriptome analyses of two freshwater stingrays (Myliobatiformes: Potamotrygonidae) from Brazil. Sci Rep 2016;6:21935.

Junqueira-de-Azevedo IL, Bastos CM, Ho PL, Luna MS, Yamanouye N, Casewell NR. Venom-related transcripts from Bothrops jararaca tissues provide novel molecular insights into the production and evolution of snake venom. Mol Biol Evol 2015;32:754-66.

Kim D, Pertea G, Trapnell C, Pimentel H, Kelley R, Salzberg SL. TopHat2: accurate alignment of transcriptomes in the presence of insertions, deletions and gene fusions. Genome Biol 2013;14:R36.

King GF, Hardy MC. Spider-venom peptides: structure, pharmacology, and potential for control of insect pests. Annu Rev Entomol. 2013;58:475-96.

King GF. Venoms as a platform for human drugs: translating toxins into therapeutics. Expert Opin Biol Ther 2011;11:1469-84.

Kordis D, Gubensek F. Adaptive evolution of animal toxin multigene families. Gene 2000;261:43-52.

Kuhn-Nentwig L, Stöcklin R, Nentwig W. Venom composition and strategies in spiders: Is everything possible? In: Casa J (ed) Advances in Insect Physiology. Academic Press, Burlignton, 2011.

Laustsen AH, Lomonte B, Lohse B, Fernández J, Gutiérrez JM. Unveiling the nature of black mamba (Dendroaspis polylepis) venom through venomics and antivenom immunoprofiling: Identification of key toxin targets for antivenom development. J Proteomics 2015;119:126-42.

Li L, Stoeckert CJ Jr, Roos DS. OrthoMCL: identification of ortholog groups for eukaryotic genomes. Genome Res 2003;13:2178-89.

Li W, Godzik A. Cd-hit: a fast program for clustering and comparing large sets of protein or nucleotide sequences. Bioinformatics 2006;22:1658-9. 
Macrander J, Broe M, Daly M. Multi-copy venom genes hidden in de novo transcriptome assemblies, a cautionary tale with the snakelocks sea anemone Anemonia sulcata (Pennant, 1977). Toxicon 2015;108:184-8.

Moura-da-Silva AM, Theakston RD, Crampton JM. Evolution of disintegrin cysteine-rich and mammalian matrixdegrading metalloproteinases: gene duplication and divergence of a common ancestor rather than convergent evolution. J Mol Evol 1996;43:263-9.

Nelsen DR, Nisani Z, Cooper AM, Fox GA, Gren EC, Corbit AG, Hayes WK. Poisons, toxungens, and venoms: redefining and classifying toxic biological secretions and the organisms that employ them. Biol Rev Camb Philos Soc 2014;89:450-65.

Olivera BM. Conus peptides: biodiversity-based discovery and exogenomics. J Biol Chem 2006;281:31173-7.

Pedroso A, Matioli SR, Murakami MT, Pidde-Queiroz G, Tambourgi DV. Adaptive evolution in the toxicity of a spider's venom enzymes. BMC Evol Biol 2015;15:290.

Pegueroles C, Laurie S, Albà MM. Accelerated evolution after gene duplication: a time-dependent process affecting just one copy. Mol Biol Evol 2013;30:1830-42.

Petras D, Heiss P, Süssmuth RD, Calvete JJ. Venom Proteomics of Indonesian King Cobra, Ophiophagus hannah: Integrating Top-Down and Bottom-Up Approaches. J Proteome Res 2015;14:2539-56.

Phuong MA, Mahardika GN, Alfaro ME. Dietary breadth is positively correlated with venom complexity in cone snails. BMC Genomics 2016;17:401.

Prashanth JR, Lewis RJ. An efficient transcriptome analysis pipeline to accelerate venom peptide discovery and characterisation. Toxicon 2015;107(Pt B):282-9.

Rachamim T, Morgenstern D, Aharonovich D, Brekhman V, Lotan T, Sher D. The dynamically evolving nematocyst content of an anthozoan, a scyphozoan, and a hydrozoan. Mol Biol Evol 2015;32:740-53.

Rendón-Anaya M, Delaye L, Possani LD, Herrera-Estrella A. Global transcriptome analysis of the scorpion Centruroides noxius: new toxin families and evolutionary insights from an ancestral scorpion species. PLoS One. 2012;7:e43331.

Reyes-Velasco J, Card DC, Andrew AL, Shaney KJ, Adams RH, Schield DR, Casewell NR, Mackessy SP, Castoe TA. Expression of venom gene homologs in diverse python tissues suggests a new model for the evolution of snake venom. Mol Biol Evol 2015;32:173-83.

Rodríguez de la Vega RC, Schwartz EF, Possani LD. Mining on scorpion venom biodiversity. Toxicon 2010;56:1155-61.

Sanggaard KW, Bechsgaard JS, Fang X, Duan J, Dyrlund TF, Gupta V, Jiang X, Cheng L, Fan D, Feng Y, Han L, Huang Z, Wu Z, Liao L, Settepani V, Thøgersen IB, Vanthournout B, Wang T, Zhu Y, Funch P, Enghild JJ, Schauser L, Andersen SU, Villesen P, Schierup MH, Bilde T, Wang J. Spider genomes provide insight into composition and evolution of venom and silk. Nat Commun 2014;5:3765. 
Smith JJ, Alewood PF. Modern venom profiling. In: Possani LD, Schwartz EF, Rodríguez de la Vega RC (eds). Scorpion Venoms. Springer, Netherlands, 2015.

Tan CH, Fung SY, Yap MK, Leong PK, Liew JL, Tan NH. Unveiling the elusive and exotic: Venomics of the Malayan blue coral snake (Calliophis bivirgata flaviceps). J Proteomics 2016;132:1-12.

Tan CH, Tan KY, Fung SY, Tan NH. Venom-gland transcriptome and venom proteome of the Malaysian king cobra (Ophiophagus hannah). BMC Genomics 2015;16:687.

Tang X, Zhang Y, Hu W, Xu D, Tao H, Yang X, Li Y, Jiang L, Liang S. Molecular diversification of peptide toxins from the tarantula Haplopelma hainanum (Ornithoctonus hainana) venom based on transcriptomic, peptidomic, and genomic analyses. J Proteome Res 2010;9:2550-64.

Trapnell C, Roberts A, Goff L, Pertea G, Kim D, Kelley DR, Pimentel H, Salzberg SL, Rinn JL, Pachter L. Differential gene and transcript expression analysis of RNA-seq experiments with TopHat and Cufflinks. Nat Protoc 2012;7:562-78.

Undheim EA, Jones A, Clauser KR, Holland JW, Pineda SS, King GF, Fry BG. Clawing through evolution: toxin diversification and convergence in the ancient lineage Chilopoda (centipedes). Mol Biol Evol 2014;31:212448.

UniProt Consortium. UniProt: a hub for protein information. Nucleic Acids Res 2015;43(Database issue):D204-12.

Utkin YN. Three-finger toxins, a deadly weapon of elapid venom--milestones of discovery. Toxicon 2013;62:50-5.

Valdez-Velázquez LL, Quintero-Hernández V, Romero-Gutiérrez MT, Coronas FI, Possani LD. Mass fingerprinting of the venom and transcriptome of venom gland of scorpion Centruroides tecomanus. PLoS One 2013;8:e66486.

van Dongen S. Graph Clustering by Flow Simulation. PhD thesis, University of Utrecht, 2000.

Verdes A, Anand P, Gorson J, Jannetti S, Kelly P, Leffler A, Simpson D, Ramrattan G, Holford M. From Mollusks to Medicine: A Venomics Approach for the Discovery and Characterization of Therapeutics from Terebridae Peptide Toxins. Toxins (Basel) 2016;8(4):E117.

von Reumont BM, Blanke A, Richter S, Alvarez F, Bleidorn C, Jenner RA. The first venomous crustacean revealed by transcriptomics and functional morphology: remipede venom glands express a unique toxin cocktail dominated by enzymes and a neurotoxin. Mol Biol Evol 2014;31:48-58.

Vonk FJ, Casewell NR, Henkel CV, Heimberg AM, Jansen HJ, McCleary RJ, Kerkkamp HM, Vos RA, Guerreiro I, Calvete JJ, Wüster W, Woods AE, Logan JM, Harrison RA, Castoe TA, de Koning AP, Pollock DD, Yandell M, Calderon D, Renjifo C, Currier RB, Salgado D, Pla D, Sanz L, Hyder AS, Ribeiro JM, Arntzen JW, van den Thillart GE, Boetzer M, Pirovano W, Dirks RP, Spaink HP, Duboule D, McGlinn E, Kini RM, Richardson MK. The king cobra genome reveals dynamic gene evolution and adaptation in the snake venom system. Proc Natl Acad Sci U S A 2013;110:20651-6. 
Xu X, Duan Z, Di Z, He Y, Li J, Li Z, Xie C, Zeng X, Cao Z, Wu Y, Liang S, Li W. Proteomic analysis of the venom from the scorpion Mesobuthus martensii. J Proteomics 2014;106:162-80.

Zhang Y, Huang Y, He Q, Liu J, Luo J, Zhu L, Lu S, Huang P, Chen X, Zeng X, Liang S. Toxin diversity revealed by a transcriptomic study of Ornithoctonus huwena. PLoS One 2014;9:e100682.

Zhang YY, Huang Y, He QZ, Luo J, Zhu L, Lu SS, Liu JY, Huang PF, Zeng XZ, Liang SP. Structural and Functional Diversity of Peptide Toxins from Tarantula Haplopelma hainanum (Ornithoctonus hainana) Venom Revealed by Transcriptomic, Peptidomic, and Patch Clamp Approaches. J Biol Chem 2015;290:14192-207. 
Table 1: Accession numbers of whole genomes and transcriptome data

\begin{tabular}{|c|c|c|c|}
\hline Species & Accession & SRAs & References \\
\hline $\begin{array}{l}\text { Acanthoscurria } \\
\text { geniculata }\end{array}$ & GCA_000671375.1 & NA & $\begin{array}{l}\text { Sanggaard et al., } \\
2014\end{array}$ \\
\hline $\begin{array}{l}\text { Centruroides } \\
\text { exilicauda }\end{array}$ & GCA_000671375.1 & NA & The i5k Initiative \\
\hline $\begin{array}{l}\text { Mesobuthus } \\
\text { martensii }\end{array}$ & GCA_000671375.1 & $\begin{array}{l}S R X 1377585^{a} \\
S R X 1376145^{b} \\
S R X 1375230^{b}\end{array}$ & Cao et al., 2013 \\
\hline $\begin{array}{l}\text { Ophiophagus } \\
\text { hannah }\end{array}$ & GCA_000516915.1 & $\begin{array}{l}\text { SRX365144 } \\
\text { SRX365143 }\end{array}$ & $\begin{array}{l}\text { Vonk, Casewell, et al., } \\
2013\end{array}$ \\
\hline
\end{tabular}

${ }^{a}$ pooled tissues; ${ }^{b}$ venom gland; NA, not available 
Table 2: Protein-coding genes summary

\begin{tabular}{|c|c|c|c|c|}
\hline \multirow[b]{2}{*}{ Species } & \multirow{2}{*}{$\begin{array}{c}\text { All gene models } \\
\text { (in paralogous } \\
\text { groups) }\end{array}$} & \multicolumn{3}{|c|}{ Toxin-like (in paralogous groups) } \\
\hline & & VZsame & VZdiff & SPbest \\
\hline $\begin{array}{l}\text { Acanthoscurria } \\
\text { geniculata }\end{array}$ & 76238 (31099) & $90 \quad(89)$ & $40 \quad(35)$ & $245(239)$ \\
\hline $\begin{array}{l}\text { Centruroides } \\
\text { exilicauda }\end{array}$ & $30465 \quad(17885)$ & $204 \quad(174)$ & $100 \quad(90)$ & $35(34)$ \\
\hline $\begin{array}{l}\text { Mesobuthus } \\
\text { martensii }\end{array}$ & 32016 (23968) & $172(143)$ & $77 \quad(72)$ & $24 \quad(23)$ \\
\hline $\begin{array}{l}\text { Ophiophagus } \\
\text { hannah }\end{array}$ & $18445 \quad(10297)$ & $68 \quad(58)$ & $13(10)$ & 270 \\
\hline
\end{tabular}

Abbreviations: VZsame, toxin-like gene models with best blast hits among VenomZone entries from the corresponding order; VZdiff, toxin-like gene models with best blast hits among VenomZone entries from a different order; SPbest, toxin-like gene models with best blast hits among proteins not known to be present in any animal venom. 
Toxin-like Gene Models

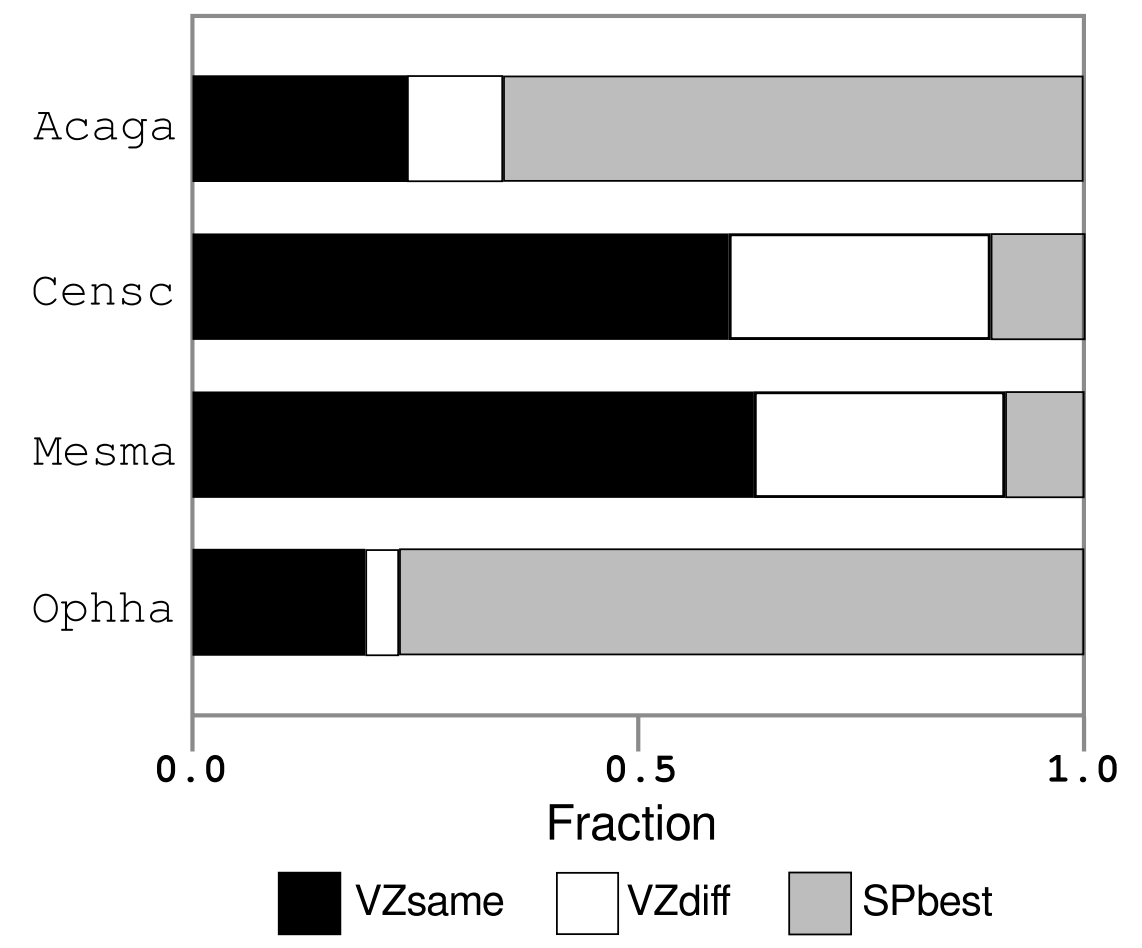



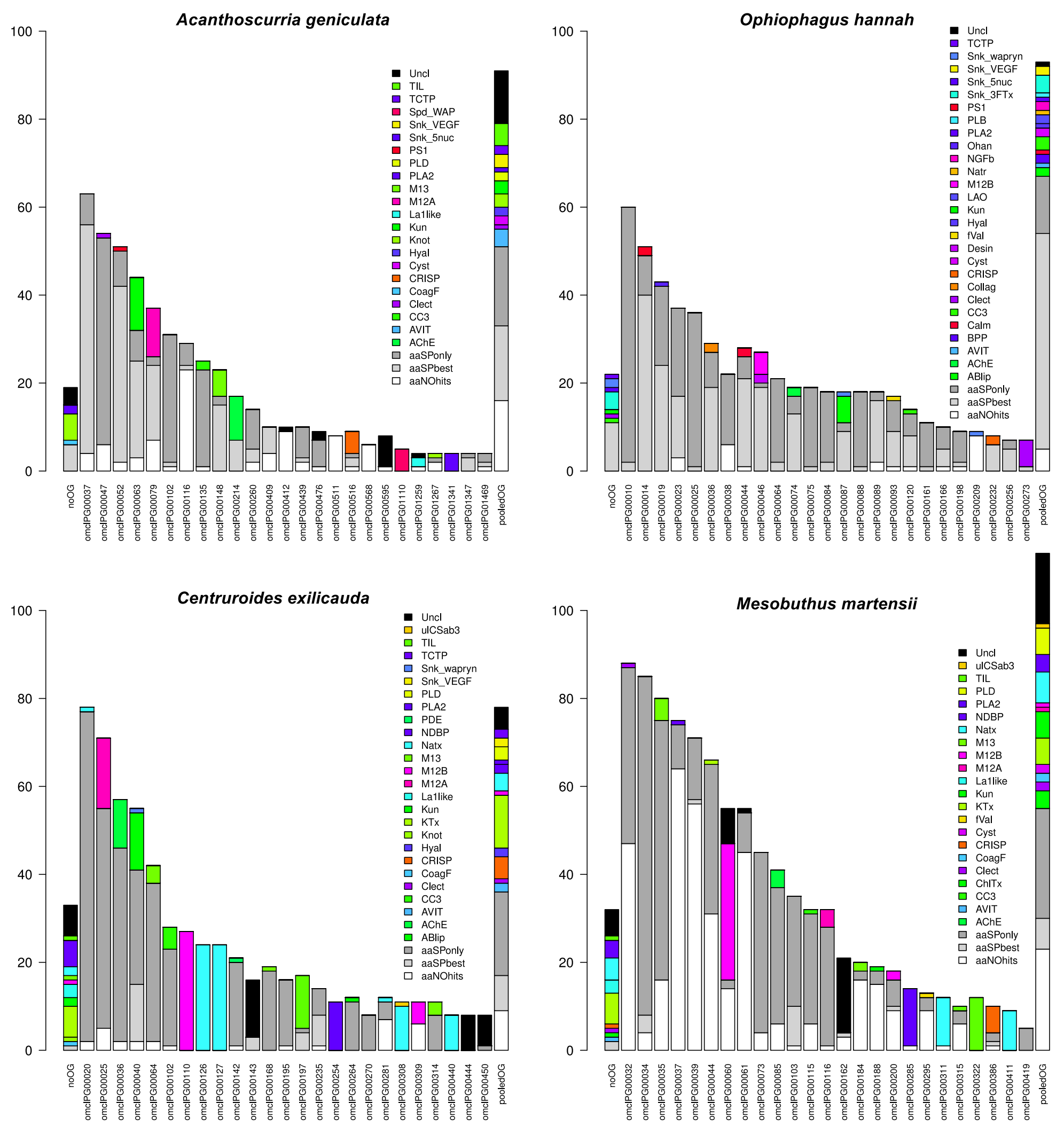

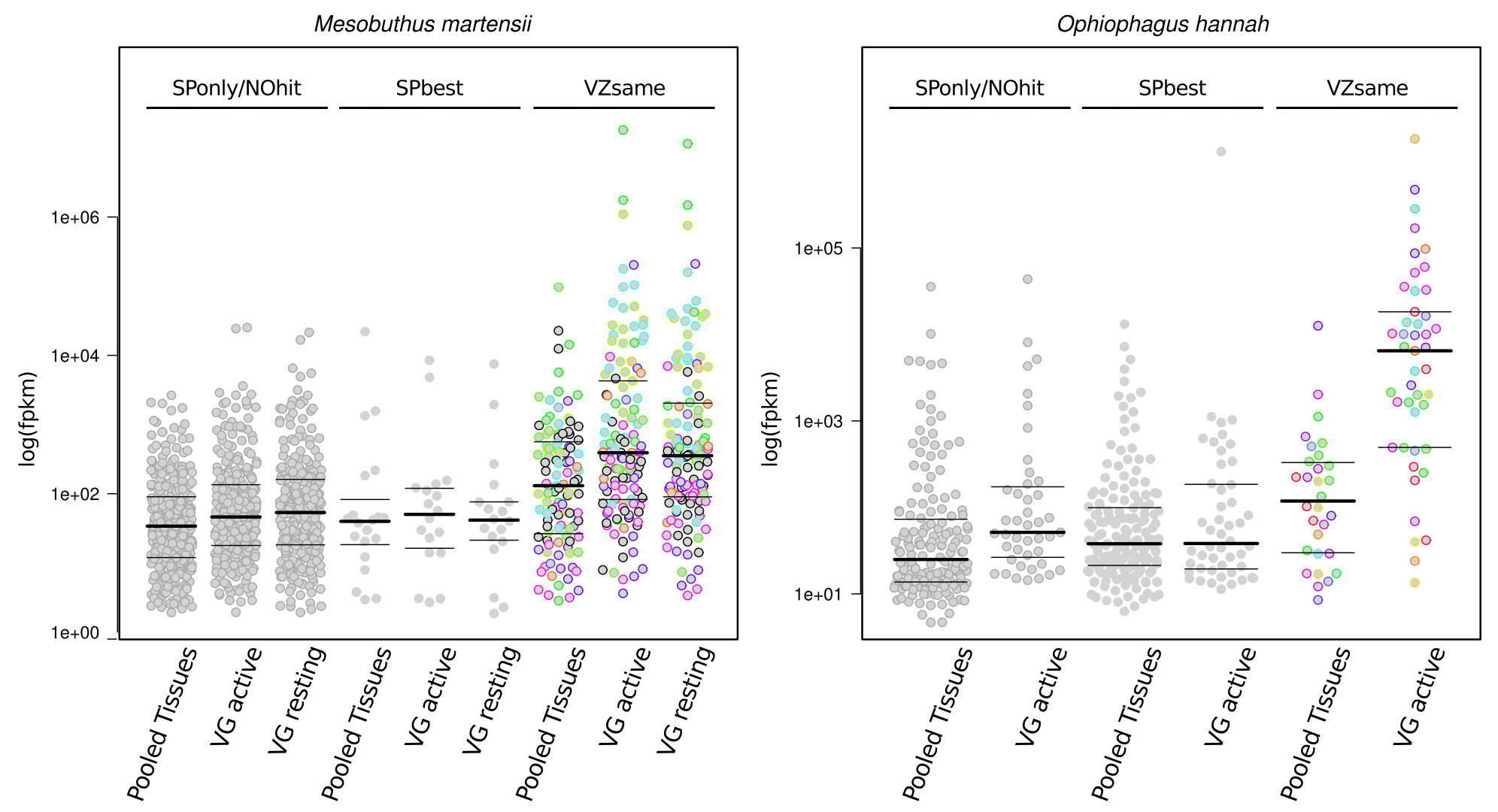